chronic lung disease, children with $\mathrm{CF} /$ chronic lung disease old enough for spirometry whose FEV1 $<50 \%$, and infants/children who required long term oxygen in the last 6 months.

This audit aimed to define which patients had undergone hypoxic challenge tests and compared this against the patient groups identified in the BTS guideline.

Methods A large tertiary children's hospital in the UK conducted a retrospective audit of hypoxic challenge tests carried out between 2012 and 2014.

Information was collected on age, diagnosis, when the patient last required long-term oxygen, lung function around the time of the test and the outcome of the test.

Results 31 HCTs from 25 patients were audited. The average age was 6.3 years (6 months-16.5 years).

Of the 22 patients who passed the HCT, three were recommended in the guideline to have HCT. Of the nine patients who did not pass HCT, two were within the recommendations.

These five patients, who were within the recommendations, were three children with $\mathrm{CF} /$ chronic lung disease with FEV1 </ $=50 \%$, one infant less than one year with history of neonatal chronic lung disease, and one child had a long term oxygen requirement within 6 months prior to testing.

Of the nine children who did not pass the HCT, seven fell outside the recommended groups. Five were ex-preterm children older than one year had history of neonatal chronic lung disease, one had SMA Type 2, one had muscular dystrophy, one had severe kyphoscoliosis and one had severe restrictive lung disease following PNET.

Conclusion Results show that clinicians are choosing to preform hypoxic challenge tests on patient outside of the recommendations. A larger proportion who failed the hypoxic challenge test were not defined within the BTS guidelines.
With advancing technology the demographics of our respiratory paediatric patient population is evolving. It is therefore prudent to reconsider the paediatric recommendations.

\section{G398(P) USE OF VIDEO, SOUND AND PULSE TRANSIT TIME TO IMPROVE ACCURACY OF SCREENING FOR OBSTRUCTIVE SLEEP APNOEA IN CHILDREN}

NJ Rowbotham, H Dove, MP Yanney. Paediatrics, Sherwood Forest Hospitals, Sutton-inAshfield, UK

\subsection{6/archdischild-2015-308599.352}

Aims Oximetry is widely used to screen for obstructive sleep apnoea (OSA) in children with good specificity but reduced sensitivity compared to polysomnography (PSG). ${ }^{1}$ Treatment decisions are usually made using oximetry criteria alone. ${ }^{2}$ Our aim is to investigate whether screening sleep studies incorporating extra modalities improve the accuracy of detecting OSA compared to oximetry alone.

Methods A retrospective review of 197 inpatient sleep studies performed at a district general hospital in 2013 was carried out using data from patient notes and a database. The sleep studies were performed with VISILAB equipment incorporating ECG, audio, video, pulse transit time (PTT) and oximetry. Study findings were categorised as 'normal'; 'primary snoring'; 'upper airway resistance syndrome' (UARS); 'obstructive sleep apnoea' or 'other abnormality'. The findings are compared with oximetry risk categories using standard criteria for OSA screening.

Results The main findings are shown in Figures 1 and 2.

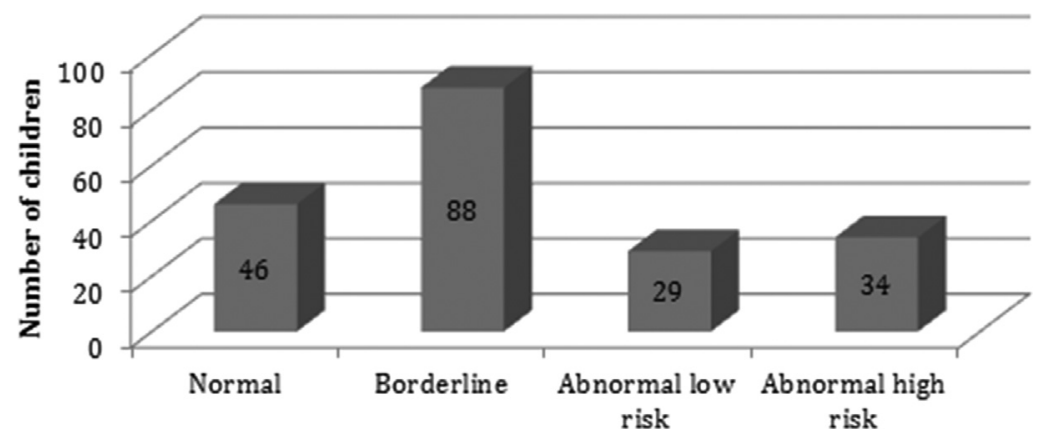

Abstract G398(P) Figure 1 Categories of risk based on oximetry data

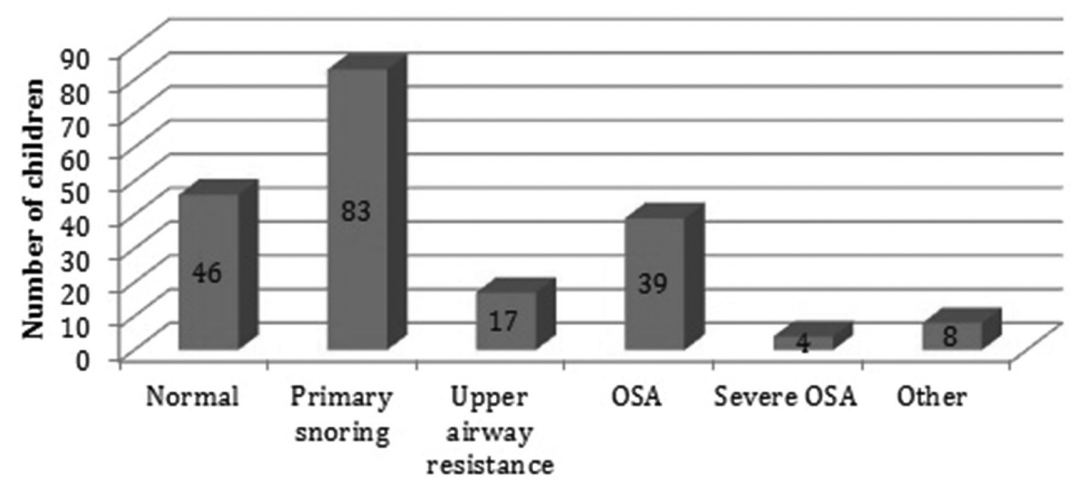

Abstract G398(P) Figure 2 Diagnosis based on sleep study findings 


\begin{tabular}{|c|c|c|}
\hline & $\begin{array}{l}\text { OSA /UARS/ other abnormality diag- } \\
\text { nosed with additional modalities }\end{array}$ & $\begin{array}{l}\text { Normal study using } \\
\text { additional modalities }\end{array}$ \\
\hline \multicolumn{3}{|c|}{ Normal or border- } \\
\hline line oximetry & 19 & 117 \\
\hline \multicolumn{3}{|l|}{ Abnormal } \\
\hline oximetry & $42^{*}$ & 19 \\
\hline
\end{tabular}

*1 child had mixed central/obstructive abnormalities.

A total of 19 children with abnormal oximetry findings were considered to have a normal study with the use of video, sound and PTT. Technical reasons were frequently identified for the abnormal oximetry findings. An identical number of children with normal or borderline oximetry were categorised as having UARS or OSA and were managed with a combination of watchful waiting, surgery or medical treatment.

Conclusion Screening sleep studies incorporating video, sound and arousal detection provide increased diagnostic accuracy over oximetry only studies. These studies may be of particular benefit in a DGH setting with no access to polysomnography.

\section{REFERENCES}

1 Brouillette RT, Morielli $A$, Leimanis $A$, et al. Nocturnal pulse oximetry as an abbreviated testing modality for pediatric obstructive sleep apnea. Pediatrics 2000;105:405-412

2 RCPCH Working Party on Respiratory Physiology and Sleep Control Disorders in Children Sept 2009

\section{G399(P) USEFULNESS OF PULSE TRANSIT TIME IN THE ASSESSMENT OF CHILDREN WITH OBSTRUCTIVE SLEEP APNOEA}

NJ Rowbotham, MP Yanney. Department of Paediatrics, Sherwood Forest Hospitals NHS Trust, Sutton-in-Ashfield, UK

\subsection{6/archdischild-2015-308599.353}

Aims Pulse oximetry is widely used to identify children with OSA but lacks sensitivity compared to polysomnography. Previous studies have shown the utility of pulse transit time (PTT) at detecting arousals in children with OSA but values likely to be indicative of disease have not been established. ${ }^{1}$ PTT is the time taken for the pulse pressure wave to travel from the aortic valve to the periphery and is a non-invasive marker of blood pressure. It provides a quantitative measurement of inspiratory effort in patients with sleep-related breathing disorders. We aim to investigate if a correlation exists between PTT indices (PTT arousals, respiratory swing) and oximetry results to ascertain its usefulness or otherwise in the assessment of children with suspected OSA.

Methods A retrospective review was carried out of 176 paediatric inpatient sleep studies undertaken at a district general hospital between Dec 2013-Dec 2014. Data were obtained from a database and patient notes. Sleep studies were carried out using VISILAB equipment incorporating ECG, audio, video, PTT and oximetry. Statistical analysis was performed using an unpaired, 1-tailed students t test.

Results Our data showed an upward trend in average PTT arousals when OSA severity was determined using pulse oximetry or by sleep study based diagnosis. Positive studies had an average PTT over 49/hour (Figures 1 and 3). This upward trend is also the case for respiratory swing (Figure 2), with positive studies having a mean respiratory swing over $24 \mathrm{~ms}$. There is a highly significant difference in mean PTT and respiratory swing

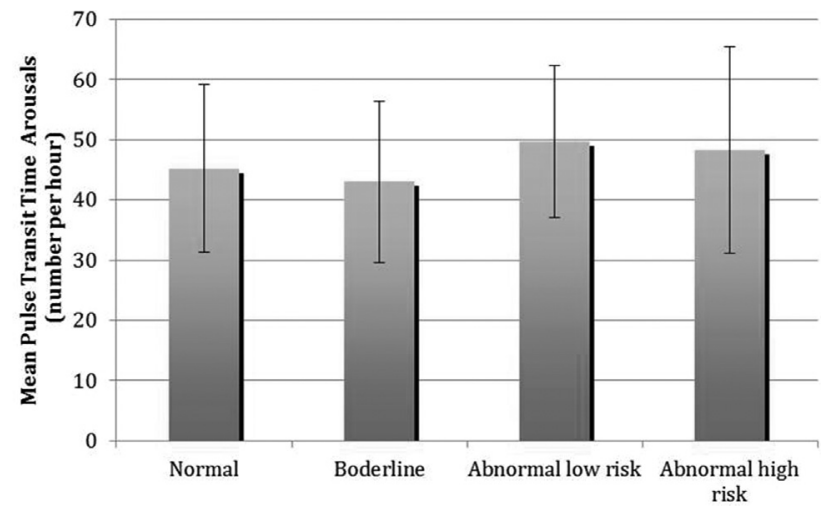

Abstract G399(P) Figure 1 Mean pulse transit time arousals for each oximetry outcome category of sleep study (error bars show standard deviation)

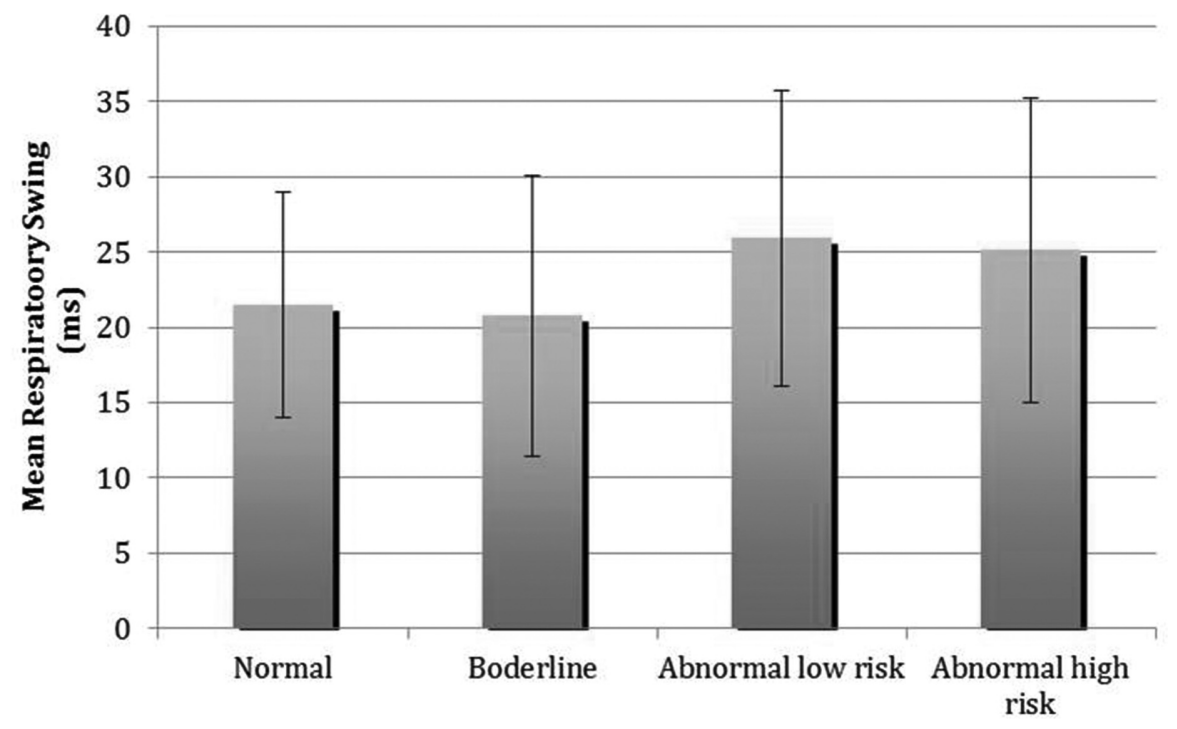

Abstract G399(P) Figure 2 Mean respiratory swing for each oximetry outcome category of sleep study (error bars show standard deviation) 